\title{
A novel strategy to prepare Ge@C/rGOhybrids as high-rate anode materials for lithium ion batteries
}

Bangrun Wang ${ }^{\mathrm{a}, \mathrm{b}}$, Zhaoyin Wen ${ }^{\mathrm{a} *}$, Jun Jin ${ }^{\mathrm{a}}$, Xiaoheng Hong ${ }^{\mathrm{a}, \mathrm{c}}$, Sanpei Zhang ${ }^{\mathrm{a}, \mathrm{b}}$, and Kun Rui ${ }^{\mathrm{a}, \mathrm{b}}$

${ }^{a}$ CAS Key Laboratory of Materials for Energy Conversion, Shanghai Institute of Ceramics, Chinese Academy of Sciences, Shanghai, 200050, P.R. China.

${ }^{b}$ Graduate School of Chinese Academy of Sciences, Beijing, 100039, P.R. China.

${ }^{c}$ Shanghaitech University, Shanghai, 200031, P.R .China

\begin{abstract}
Germanium is considered as a promising anode material for lithium ion batteries (LIBs) due to its high-capacity. However, owing to the huge volume variation during cycling, the batteries based on germanium anodes usually show poor cyclability and inferior rate capability. Herein, wedemonstrated a novelstrategy to uniformly anchor the core-shell structured germanium@carbon (Ge@C)onthereduced graphene oxide (rGO) nanosheetsby the strong adhesion of dopamine. In the resulting Ge@C/rGO hybrid, the amorphous carbon layerandrGO nanosheets can effectively reduce the agglomeration of germanium and provide buffer matrix for the volume change in electrochemical lithium reactions. When used as anode materials for LIBs, Ge@C/rGO hybrids deliver a reversible capacity of $1074.4 \mathrm{mAh} \mathrm{g}^{-1}$ at $2 \mathrm{C}$ after 600 cycles (with capacity retention of 96.5\%) and high rate capability of $436 \mathrm{~mA} \mathrm{~h} \mathrm{~g}^{-1}$ at $20 \mathrm{C}$ after 200 cycles. The encouraging electrochemical performance clearly demonstrates that Ge@C/rGO hybrids could be a potential anode material with high capacity, excellent rate capability, and good cycling stability for LIBs.
\end{abstract}

Keywords: Lithium-ion battery; germanium anode; double carbon protection; strong adhesion; dopamine.

Corresponding author. Tel: +86-21-52411704, Fax: =86-21-52413903

Email address: zywen@mial.sic.ac.cn

(C) 2016. This manuscript version is made available under the Elsevier user license http://www.elsevier.com/open-access/userlicense/1.0/ 


\section{Introduction}

Over the past two decades, lithium ion batteries (LIBs)have been widely used as energy-storage systems for portable electronic devices, such as mobile phones and laptops. With the development of electric vehicles (EVs) and hybrid electric vehicles (HEVs), the next-generation LIBs with higher energy and power densities are needed to be progressed[1-4]. Currently, the commercial graphite anode cannot meet the requirement of high energy density due to its low theoretical capacity (372 $\mathrm{mAh} \mathrm{g}^{-1}$ ). Therefore, numerous efforts have been proposed in search of alloy-type anodes that have been considered as the most promising anode candidates for the next-generation LIBs because of theirgreat abundance, moderate working potential and high theoretical capacities (4200 $\mathrm{mAh} \mathrm{g}^{-1}$ for silicon, $1600 \mathrm{mAh} \mathrm{g}^{-1}$ for germanium)[5-8]. However, their practical utilization is hindered by the large volume change caused by alloying process[9]Silicon can alloy with lithium to form $\mathrm{Li}_{4.4} \mathrm{Si}$ along with the huge volume change up to $300-400 \%$ that causes the pulverization of active materials and instability of the solid electrolyte interphase (SEI) films, leading to poor electrochemical performance[10, 11].Compared to silicon, germanium exhibits faster lithium diffusivity (400 times more than $\mathrm{Si}$ ) and superior electrical conductivity (104 times more than Si) [12-14]. Nevertheless, similar to silicon,germanium experiences large volume changes (over 300\% after full lithiation) during the lithiation/delithiation process and the stress that result from the cycling process can lead to the pulverization, cracking and destabilization SEI films of germanium electrodes. These issues cause rapid capacity fading upon cyclingand low coulombic efficiency, which hinder the practical application of germanium anodes[13, 15, 16].

Several strategies have been adopted to buffer the volume change of germanium during 
cycling, including making use of nanowires [17, 18], nanotubes [19], and porous germanium [20]. In addition, carbon coating is a common strategy for germanium anodes. During the cycling process, carbon not only acts as a buffer matrix to suppress the large volume changedue to its favorable thermodynamic stability, but also work as a conductive material to enhance the electrical conductivity because of its high electronic conductivity. Furthermore, carbon matrix contributes to improving the lithium ion conductivity,thereby improving the electrochemical performance of germanium anodes[21-29].Among the carbonaceous materials, graphene, with superior electrical conductivity, excellent mechanical flexibility and good chemical stabilityhas been extensively studied as a conducting and buffering matrix in LIBs [7, 21, 30-32]. Recently, a double carbon protection strategy has been proposed, in which germanium is protected by amorphous carbon and graphene. In related work, Guo et al.reported Ge@C/RGO composites by simply distributing Ge@C on the RGO sheets,exhibiting a reversible capacity of $~ 940$ mAh g ${ }^{-1}$ at a current density of $50 \mathrm{~mA} \mathrm{~g}^{-1}$ after 50 cycles [21]. Nevertheless, the rate capability and cycling stability are still remained to be improved. Therefore, it is desirable to choose a carbon source that has a strong adhesion with germanium and graphene to reduce the agglomeration of germanium and ameliorate structural stability, which is the key to double carbon protection strategy. In this regard, dopamine is a potentialcarbon source due to its special properties. On one hand, dopamine can self-polymerize at alkaline/neutral $\mathrm{pH}$ values to form a continuous and homogeneously carbon shell under oxygen atmosphere [33-38]. On the other hand, dopamine has a strong adhesion to a wide range of organic and inorganic substances under acid $\mathrm{pH}$ values owing to its derivatives of catecholic(a mussel-inspired process)[34, 39].Dopamine was not only adopted as the carbon precursor but it also played an important role in further protecting germanium from pulverization. 
In this work, we designed a double carbon protection strategy for germanium, in whichcore-shell structuredgermanium@carbon (Ge@C) compositeswere homogeneously anchored on the reduced graphene oxide nanosheets (Ge@C/rGO).Germanium wasfirst prepared by a simple mechanochemical reaction using $\mathrm{GeO}_{2}$ and $\mathrm{Mg}$ powders. Then, germanium particles were coated with polydopamine films to form the core-shell structured germanium@polydopamine (Ge@PDA) composites, which then were uniformly anchored on the graphite oxide (GO) through the strong adhesion of dopamine followed by the subsequent calcination to form the Ge@C/rGO hybrids (Figure 1). The as-prepared Ge@C/rGO hybrids show high specific capacity, excellent cycling performance and outstanding rate capability in comparison with Ge@C composites as anode materials inLIBs. In view of the fact that the present strategy is simple and effective, itcould also be applied to other alloy-type anode materials that have large volume change and low electronic conductivity.

\section{Experimental}

\subsection{Materials}

Germanium dioxide $\left(\mathrm{GeO}_{2}\right.$, 99.999\%) provided by Germanium Co., Ltd. (China), Magnesium power (Mg, 99\%, 100 200 mesh) provided by Sinopharm Chemical Reagent Co., Ltd. (China), Tris(hydroxymethyl) aminomethane, Dopamine hydrochloride $\left(\mathrm{C}_{8} \mathrm{H}_{11} \mathrm{NO}_{2} \cdot \mathrm{HCl}\right)$ and Graphite powder (99.95\%, 8000 mesh) supplied by Aladdin Chemistry Co., Ltd. (China), were used as received.

\subsection{Synthesis of Ge and Ge@C/rGO hybrid}

Germanium was prepared by mechanochemical reaction of $\mathrm{GeO}_{2}$ and $\mathrm{Mg}$ powders followed by an etching process with hydrochloric acid $(\mathrm{HCl})$ solution. Briefly, $4 \mathrm{~g} \mathrm{GeO}_{2}$ and $2 \mathrm{~g} \mathrm{Mg}$ powders 
were mixed completely and ground to form a uniform gray mixture, which was sealed into a steel bowl in an Ar-filled glove box. After high energy mechanical milling (HEMM) of the mixture at a rotation speed of $400 \mathrm{rpm}$ for 3 hours on a Planetary Mill P-5 (Fritsch, Germany), the obtained black powders were immersed in $1 \mathrm{M} \mathrm{HCl}$ solution for 4 hours with stirring to remove the by-product MgO. Thenproducts were washed by centrifugation with deionized water and dried at $60{ }^{\circ} \mathrm{C}$ in a vacuum oven for 12 hours.

$200 \mathrm{mg}$ of germanium powders were dispersed in $100 \mathrm{ml}$ deionized water by ultrasonication for 30 minutes. Then $1 \mathrm{mmol}$ of tris (hydroxymethyl) aminomethane $(10 \mathrm{mM}, \mathrm{pH}=8.5)$ and $1 \mathrm{mmol}$ dopamine hydrochloride were added to the resulting dispersion. After stirring for 24hours, the Ge@PDA products were washed by centrifugation with deionized water three times.Graphite oxide (GO) dispersion was synthesized through a modified Hummers method [40]. 100mL of GO dispersion (1mg mL ${ }^{-1}$ ) was mixed with 400 mg of core-shell structured Ge@PDA composites by ultrasonication for 2 hours. Then $40 \mathrm{mg}$ of dopamine was added to the above mixture at $\mathrm{pH}=3$ [39]. After stirring for 24 hours, the Ge@PDA/GO composite was collected by centrifugation with deionized water three times and then subjected to freeze-drying process for 24 hours.The obtained Ge@PDA/GO composites were sintered in a tube furnace at $700{ }^{\circ} \mathrm{C}$ for 2 hours under $\mathrm{Ar}$ (95 v\%)/ $\mathrm{H}_{2}$ (5 v\%) atmosphere, the heating rate was kept at $2^{\circ} \mathrm{Cmin}^{-1}$. Ge@C/rGOhybrids were obtained after cooling to room temperature.

\subsection{Characterizations and Electrochemical tests}

The morphology of the as-prepared materials and the electrode surface were investigated by a TEM (JEM-2100F) and a SEM (S-4800N, Hitachi), respectively. XRD (Rigaku Ultima IV, $40 \mathrm{KV} / 20 \mathrm{~mA}, \mathrm{Cu}-\mathrm{K} \alpha$ radiation) was used to characterize the phases of the as-prepared materials. 
HRTEM and SAED measurements were also performed using a JEM-2100F transmission electron microscope. The carbon content in the composite was detected using thermogravimetric analyzer (Netzsch STA 409PC) under air atmosphere from room temperature to $1000^{\circ} \mathrm{C}$ at a heating rate of $5^{\circ} \mathrm{Cmin}^{-1}$.

Electrochemical measurements were performed using CR2025 coin-type cells assembled in an Ar-filled glove box with oxygen and water contents less than $1 \mathrm{ppm}$. The working electrode consisted of active material (Ge@C/rGO, Ge@C and Ge), super P, and lithium polyacrylate (Li-PAA) mixed in a weight ratio of 8:1:1, respectively. Deionized (DI) water was added to form homogeneous slurry. Then, the resultant slurry was uniformly coated on a Cu foil current collector followed by drying in vacuum at $80^{\circ} \mathrm{C}$ for 24 hours, and the electrode was cast into 12 mm-diameter disks. Electrochemical cells were assembled with the cast electrode, Li metal as both counter and reference electrodes, and glass fiber (Whatman) was employed as the separator. The electrolyte involves $1 \mathrm{M} \mathrm{LiPF}_{6}$ in ethylene carbonate (EC), dimethyl carbonate (DMC) (1:1 by volume), and $5 \mathrm{wt} \%$ fluoride ethylene carbonate (FEC). The average mass loading of the active material on each electrode was about $0.8 \sim 1.0 \mathrm{mg}$. Galvanostatic charge-discharge measurements of the electrodes were performed at room temperature in the potential range $0.01 \sim 1.5 \mathrm{~V} \mathrm{vs.} \mathrm{Li}^{+} / \mathrm{Li}$ using a LANDCT2001A battery test system. Cyclic voltammogram measurements were measured on an electrochemical workstation (CHI700C) at a scan rate of $0.1 \mathrm{mV} \mathrm{s}^{-1}$ in the same range of 0.01 1.5V. Electrochemical impedance spectroscopy (EIS) measurements in the frequency range of $0.01-10^{5} \mathrm{~Hz}$ with AC amplitude of $10 \mathrm{mV}$ were carried out on an Autolab PGSTAT302 potentiostat-galvanostata electrochemical work station (Metrohm).

\section{Results and discussion}


The overall synthesis route of Ge@C/rGO is depicted schematically in Figure 1.Germanium was prepared by mechanochemical reaction of $\mathrm{GeO}_{2}$ and $\mathrm{Mg}$ powders, followed by an etching process (Figure 1a)[41]. Then germanium powders were coated with a homogeneous layer of polydopamine (PDA) to form a core-shell structured Ge@PDA composites. The as-synthesized core-shell structured Ge@PDA particles were immobilized on the graphite oxide (GO) in the presence of dopamine at $\mathrm{pH}=3$ [39].GO was converted to reduced graphene oxide ( $\mathrm{rGO}$ ) after calcination at $700{ }^{\circ} \mathrm{C}$, and core-shell structuredGe@C were anchored on the rGO nanosheets to form Ge@C/rGO hybrids (Figure 1b).

a
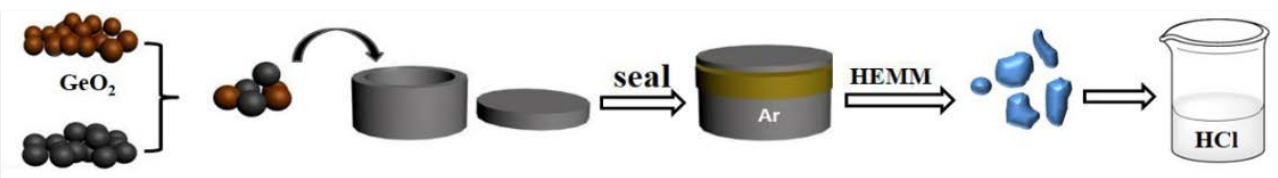

Mg
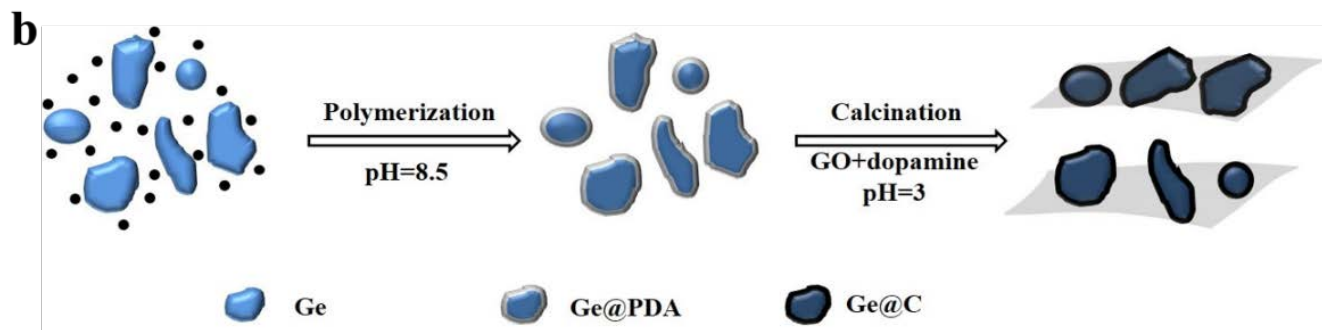

Ge@PDA

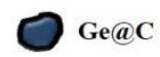

- dopamine

reduced graphene oxide (rGO)

Figure 1.Schematic illustration of the synthesis route for (a) Ge and (b) Ge@C/rGO.

a

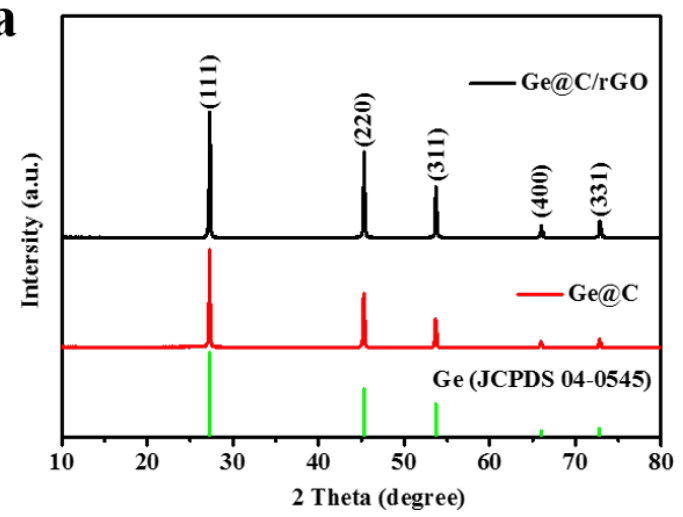

b

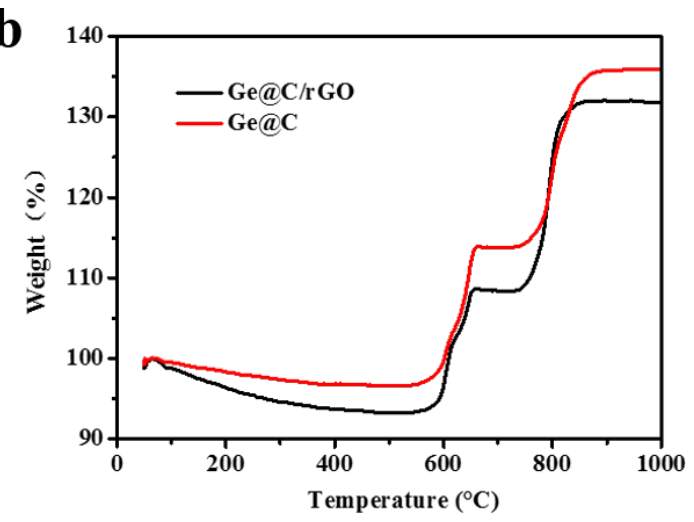

Figure 2.(a) Powder X-ray diffraction patterns and(b) Thermogravimetric analysis curves of Ge@C and Ge@C/rGO. 
The crystal structure of the $\mathrm{GeO}_{2}$ precursor, germanium, Ge@C,and Ge@C/rGO hybrids were determined by powder X-ray diffraction (XRD), as shown in Figure 2a and Figure S1 (Supporting Information). Main diffraction peaks at $2 \theta$ values of $27.3^{\circ}, 45.3^{\circ}$, and $53.7^{\circ}$ in the XRD patterns of Ge@C and Ge@C/rGO products can be well assigned to the (111), (220), and (311) planes of diamond cubic phase of Ge (joint committee on powder diffraction standard (JCPDS) card no. 04-0545) [4]. From the XRD patterns of both Ge@C and Ge@C/rGO products, no carbon peaks can be detected, which may be attributed to the high intensity of the (111) peak of germanium and the disordered nature of carbon. The Raman spectroscopy of Ge@C/rGO hybrids (as shown in Figure S2, Supporting Information) can confirm the existence of carbon and germanium in the Ge@C/rGO composites. The peak located at $298 \mathrm{~cm}^{-1}$ in Ge@C/rGO composites corresponding to the Ge-Ge phonon vibration [42, 43]. The two typical Raman peaks at 1345 and1581 $\mathrm{cm}^{-1}$ can be indexed to the D-band and G-band of carbon, respectively [44, 45]. Therefore, the carbon contents of Ge@C and Ge@C/rGO products can be detected through thermogravimetric analysis (TGA) (Figure 2b). On the basis of the mass increase of Ge@C andGe@C/rGO products, the carbon content of Ge@C and Ge@C/rGO products are calculated to be $\sim 5.7 \mathrm{wt} \%$ and $\sim 8.4 \mathrm{wt} \%$, respectively $\left(\mathrm{GeO}_{2}\right.$ was supposed to be the final product) [22]. 

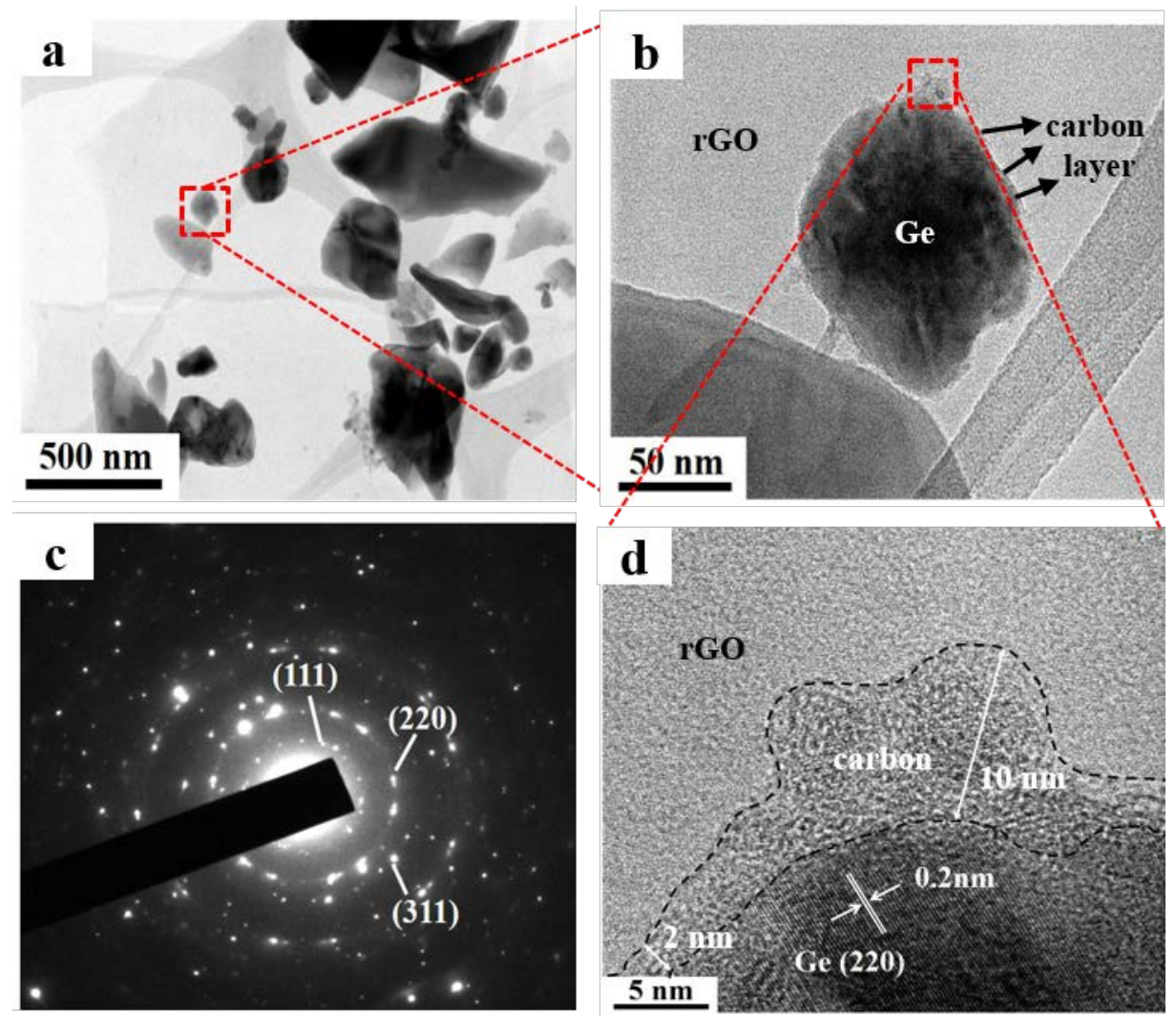

Figure 3. (a-b) TEM images;(c) SAED; (d) HRTEM image of Ge@C/rGO.

The microstructures of Ge@C/rGO hybrids were characterized by transmission electron microscopy (TEM) (Figure 3a and b). Ge@C particles are found to be anchored on the rGO sheetsand uniformly distributed from low magnification TEM image (Figure 3a).The TEM images of germanium and Ge@C products are shown in Figure S3 (Supporting Information).In the high-resolution TEM image (Figure 3d), the germanium is coated with the amorphous carbon and graphene, and the crystal lattice fringes with a d-spacing of $0.2 \mathrm{~nm}$ are indexed to the (220) lattice planes of cubic germanium. The thin and amorphous carbon layer can be clearly seen with a thickness of 2-10 $\mathrm{nm}$. The selected-area electron diffraction (SAED) pattern also reveals that the as-synthesized germanium has a diamond cubic structure in the Ge@C/rGO hybrids (Figure 3c). The d-spacing values calculated from the SAED pattern were $0.33,0.2$, and $0.17 \mathrm{~nm}$, consistent with the d-spacing values for the (111), (220), and (311) planes of diamond cubic phase of Ge, 
respectively [21]. Ge@C composites are uniformlyanchored onto the surface of rGO nanosheets, which indicating that carbon layers and graphene nanosheets can form a hybrid conducting and buffering matrix for germanium.
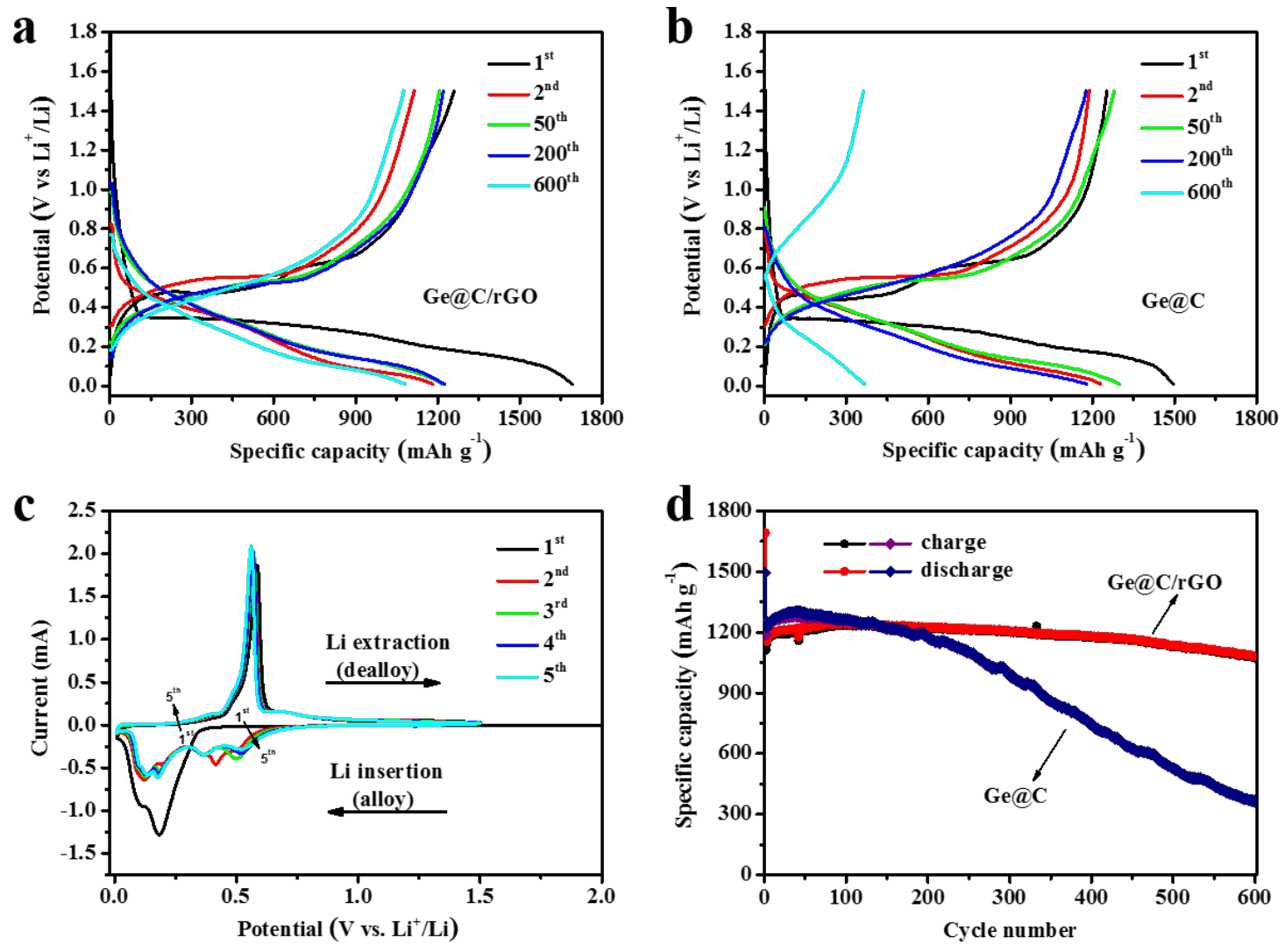

Figure 4. (a-b) Voltage profiles of the Ge@C/rGO and Ge@C electrodes, the rate was C/10 for the first cycle, then 2C for the later cycles; (c) Cyclic voltammograms of Ge@C/rGO corresponding to the first five cycles at a scan rate of $0.1 \mathrm{mV} \mathrm{s}^{-1}$; (d) Cycling performanceof the Ge@C/rGO and Ge@C electrodes.

The electrochemical performance of the Ge@C and Ge@C/rGO electrodes as anode materials for lithium ion batteries were investigated by galvanostatic discharge-charge. Figure 4ashows the discharge-charge voltage profiles of the Ge@C/rGO electrode at a low rate of C/10 (the current density of $1 \mathrm{C}$ rate is $1600 \mathrm{~mA} \mathrm{~g}^{-1}$ ) for the first cycle, followed by $2 \mathrm{C}$ rate for the subsequent cycles over the voltage range $0.01-1.5 \mathrm{~V} \mathrm{vs} . \mathrm{Li}^{+} / \mathrm{Li}$. The specific capacities were 
calculated based on the total weight of composites, i.e. Ge@C, Ge@C/rGO[21, 23]. In the initial discharge process, there is a voltage plateau around $0.3 \mathrm{~V}$ because of the lithium alloying with germanium to form $\mathrm{Li}_{\mathrm{x}} \mathrm{Ge}$ alloy. In the following discharge process, the plateau is replaced by a long slope. About the first charge process, it has two delithiation platforms below $0.7 \mathrm{~V}$, which is similar to the reported literature $[15,46]$. The initial discharge and charge specific capacities are 1692.3 and $1258.5 \mathrm{mAh} \mathrm{g}^{-1}$ at $\mathrm{C} / 10$, respectively, corresponding to initial coulombic efficiency (ICE) of $74.4 \%$. The low ICE is mainly ascribed to decomposition of electrolyte to form solid electrolyte interphase (SEI) films on the electrode surface, in addition to the irreversible lithium insertion into the composites during the first discharge step [13, 26, 47, 48]. After the second cycle, the CE of Ge@C/rGO electrode was significantly improved to >98\% for up to 600 cycles. Under the same conditions, the Ge@C shows an initial discharge and charge capacity of 1495.3 and 1252.1 $\mathrm{mAh} \mathrm{g}^{-1}$, respectively (Figure $4 \mathrm{~b}$ ), corresponding to ICE of $83.7 \%$. The $\mathrm{N}_{2}$ adsorption measurements of Ge@C and Ge@C/rGO products (as shown in Figure S4, Supporting Information). The specific surface area of $\mathrm{Ge} @ \mathrm{C}$ and $\mathrm{Ge} @ \mathrm{C} / \mathrm{rGO}$, calculated by the Brunauer-Emmett-Teller (BET) method, are about 1.29 and $278.96 \mathrm{~m}^{2} \mathrm{~g}^{-1}$, respectively. The Ge@C/rGO electrode with higher specific surface area can increase the contact area between the graphene and electrolyte, leading to more surface side reactions, and therefore more SEI films and higher reversible lithiumcapacity loss. In consequence, the ICE of Ge@C/rGO electrode is lower than that of the Ge@C electrode [4, 49-52].Figure 4c shows the typical cyclic voltammograms (CVs) of the Ge@C/rGOelectrode in the initial five cycles at a scan rate of $0.1 \mathrm{mV} \mathrm{s}^{-1}$ in the potential range $0.01-1.5 \mathrm{~V}$ vs. $\mathrm{Li}^{+} / \mathrm{Li}$. During the first lithiation step, a sharp cathodic peak was centered at $0.18 \mathrm{~V}$, which is attributed to the amorphization of crystalline germanium due to the 
lithiation. The sharp peak is accompanied by a broad peak at $0.11 \mathrm{~V}$, which is related to the formation of amorphous $\mathrm{Li}_{\mathrm{x}} \mathrm{Ge}$ because of the further lithium insertion of amorphous germanium[46, 53].There are no obvious peaks about the formation of SEI, indicating a less irreversible lithium loss by reason of the side reaction [54]. However, the sharp peak at $0.18 \mathrm{~V}$ was substituted by a series of broad peaks on account of the lithium alloying with amorphous-Ge from the second cycle. For the delithiation process, the main two peaks at 0.56 and $0.67 \mathrm{~V}$ corresponded to delithiation of $\mathrm{Li}_{\mathrm{x}} \mathrm{Ge}$ alloys [53, 54]. Figure 4d shows the cycling performance of the Ge@C and Ge@C/rGO electrodes. The capacity of the Ge@C/rGO electrode slowly increase to 1243.6 $\mathrm{mAh} \mathrm{g}^{-1}$ at the 100th cycle, which can be attributed to the electrochemical activation process. After discharging-charging for 600 cycles, the Ge@C/rGO electrode exhibits a reversible capacity of $1074.4 \mathrm{mAh} \mathrm{g}^{-1}$, which is $96.5 \%$ of the capacity at the 2nd cycle. And no obvious capacity fading is observed during cycling. In contrast, the reversible capacity of the Ge@Celectrode decreases from 1252.1 to $362.6 \mathrm{mAh} \mathrm{g}^{-1}$ continuously after discharging-charging for 600 cycles, which indicates that the only amorphous carbon layers cannot guarantee stable long-term cycling performance. In addition, it can also be observed that the Ge@Celectrode exhibits relatively high reversible capacity in the first 150 cycles. The reason may be attributed to its relatively low carbon content, which increases the germanium content (with high theoretical capacity) in the resultant composite. The rapid capacity fading is mainly because of low electronic conductivity and the severe volume change of germanium in the cycling process leading to the loss of active material. The cycling performance and discharge-charge voltage profiles of germanium electrode (as shown in Figure S5, Supporting Information) shows rapid fading of capacity with cycles increased. The scanning electron microscopy (SEM) of Ge@C and Ge@C/rGO electrodes surface (as shown in 
Figure S6, Supporting Information) after discharging-charging for 600 cycles. As shown in Figure S6a, the Ge@C electrode suffers from severe pulverization and most of the active materials even lose contact with the current collector, indicating the structural destruction of Ge@C materials after cycling. The amorphous carbon layer may crack due to the large volume change of Ge. In contrast, the cycled Ge@C/rGO electrode maintains better surface morphology from which only a few active materials fall off the current collector (Figure S6b). The high elasticity of rGO can buffer the stress generated during the lithiation/delithiation of Ge since the Ge@C particles are anchored onto the surface of rGO sheets in the Ge@C/rGO hybrids, which is conductive to protect the integrity of carbon shells. Therefore, the Ge@C/rGO electrode exhibits better structural stability than the Ge@C electrode.

The TEM images of Ge@C/rGO materials after 600 cycles are shown in Figure S7 in order to further demonstrate its high structural stability. As displayed in Figure S7a, the structural integrity of some Ge@C particles (the dark ones) was maintained without pulverization owing to the high elasticity of rGO matrix, the particle size (nm) is similar to the one before cycling (Figure 3a). As observed, some pristine Ge particles transformed to fine nanoparticles after 600 cycles, resulting in many small Ge particles dispersed on the rGO surface. Although the rGO sheets are not very clearly displayed in the low magnification TEM image owing to either its low content in the Ge@C/rGO hybrids orthe organic binder on the surface of the active materials. However, the diffraction fringes of rGO can be clearly observed in the HRTEM image (Figure S7b)to prove its existence.The function of the rGO nanosheets in the germanium-based anodes to improve the structural stability and electrochemical cycle performance were demonstrated. 

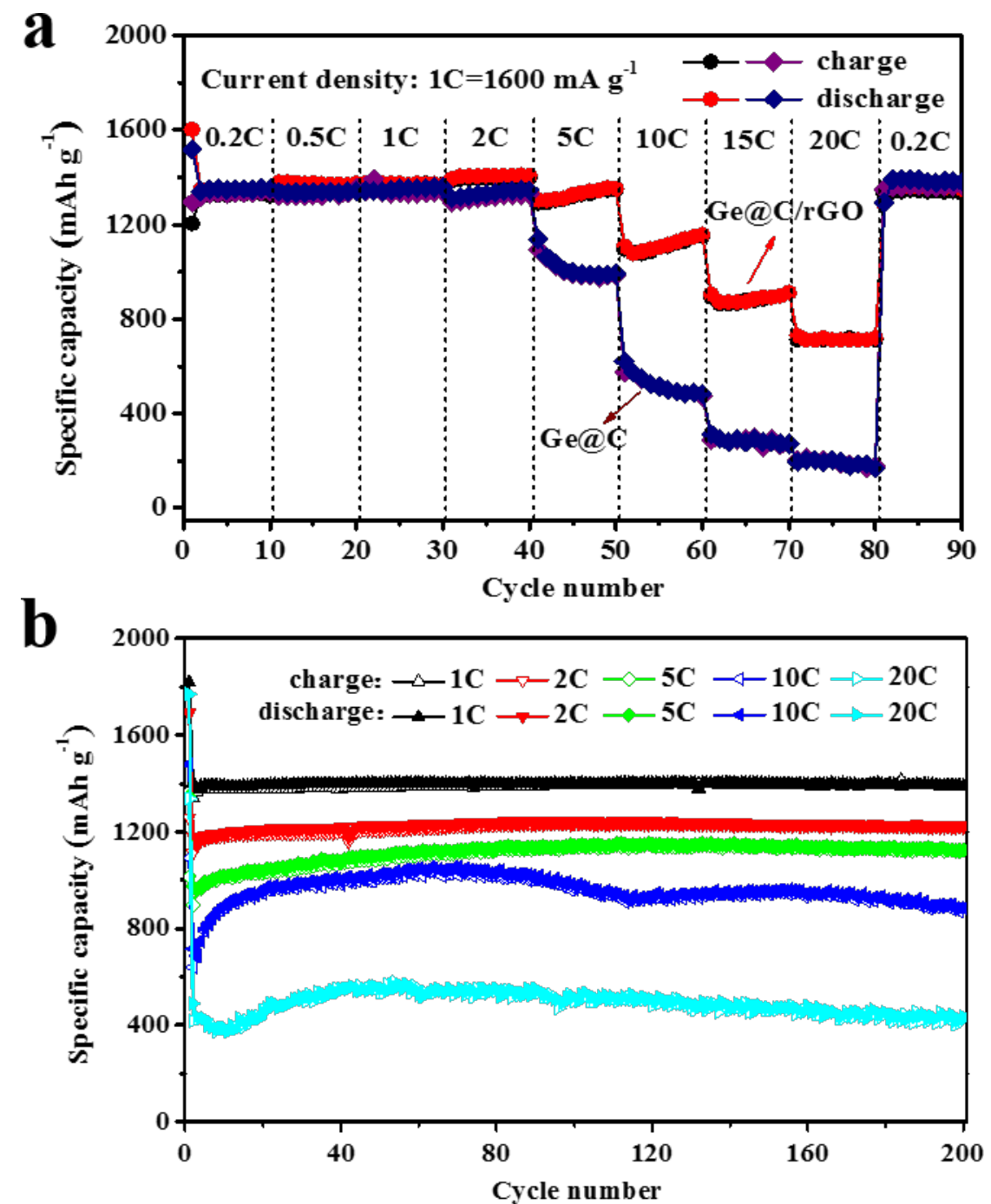

Figure 5.(a) Rate capability of Ge@C/rGO and Ge@C electrodes; (b) Discharge and charge capacity as a function of cycle numbers at different rates (after activation, $0.1 \mathrm{C}$ for the first cycle) of Ge@C/rGO electrode.

The rate capability of Ge@C/rGO and Ge@C electrodes were further tested at different current densities (Figure 5a). The Ge@C/rGO electrode shows much better rate capability than the Ge@C electrode. With the increase of discharge/charge current rates (from 2C to 20C), the improvement of reversible capacities became remarkable. At the rates of 5C, 10C, and 15C, the Ge@C/rGO electrode delivers reversible capacities of 1319.0 (45th), 1104.1 (55th), and 879.4 mAh $g^{-1}$ (65th), respectively. Under the same current densities, the Ge@C electrode shows 
reversible capacities of 988.9 (45th), 511.0 (55th), and $293.1 \mathrm{mAh} \mathrm{g}^{-1}$ (65th), respectively. Even at a high rate of 20C, the Ge@C/rGO electrode can also maintain reversible capacity of 711.6 mAh $\mathrm{g}^{-1}$ (75th). However, the Ge@C electrode can only show reversible capacity of $204.3 \mathrm{mAh}$ $\mathrm{g}^{-1}$ (75th) at the current rate of 20C. Furthermore, both electrodes recovered high capacity when the rate was decreased to 0.2C, indicating good reversibility of Ge@C/rGO and Ge@C products as anode materials in LIBs. The discharge/charge voltage profiles of Ge@C/rGO and Ge@C electrodes (as shown in Figure S8 (a) and (b), Supporting Information) in the range of 0.01-1.5 V at different current rates, respectively. The Ge@C/rGO and Ge@C electrodes show obvious voltage plateau at $\sim 0.2 \mathrm{~V}$ in the discharge profiles and $\sim 0.5 \mathrm{~V}$ in the charge profile, corresponding to the alloying and dealloying of $\mathrm{Li}_{x} \mathrm{Ge}$, respectively.The rate capability and discharge/charge voltage profiles of germanium electrode are as shown in Figure S8 (c) and (d) (Supporting Information), in which the reversible capacity of germaniumelectrode is $\sim 0 \mathrm{mAh} \mathrm{g}^{-1}$ at the $20 \mathrm{C}$ rate.The cycling stability of the Ge@C/rGO electrode was also characterized at various discharge/charge current rates, the result is presented in Figure 6b. After lithiation-delithiation for 200 cycles, no obvious drop of capacity can be observed with these stabilized capacity-cycle number curves from 1 to 10C rate. When the discharge/charge rate was elevated up to 20C, the specific capacity of Ge@C/rGOelectrode slightly decreased to $436 \mathrm{mAh} \mathrm{g}^{-1}$, this value was still much higher than the theoretical capacity of graphite anode $\left(372 \mathrm{mAh} \mathrm{g}^{-1}\right)$.The excellent rate performance of the Ge@C/rGO electrode is probably attributed to the double carbon matrix composed of amorphous carbon and rGO nanosheets. The carbon layers can offer enough conductive pathways for electron lithium ion diffusion. Furthermore, less agglomeration of Ge@C particles can increase the utilization of active materials. 

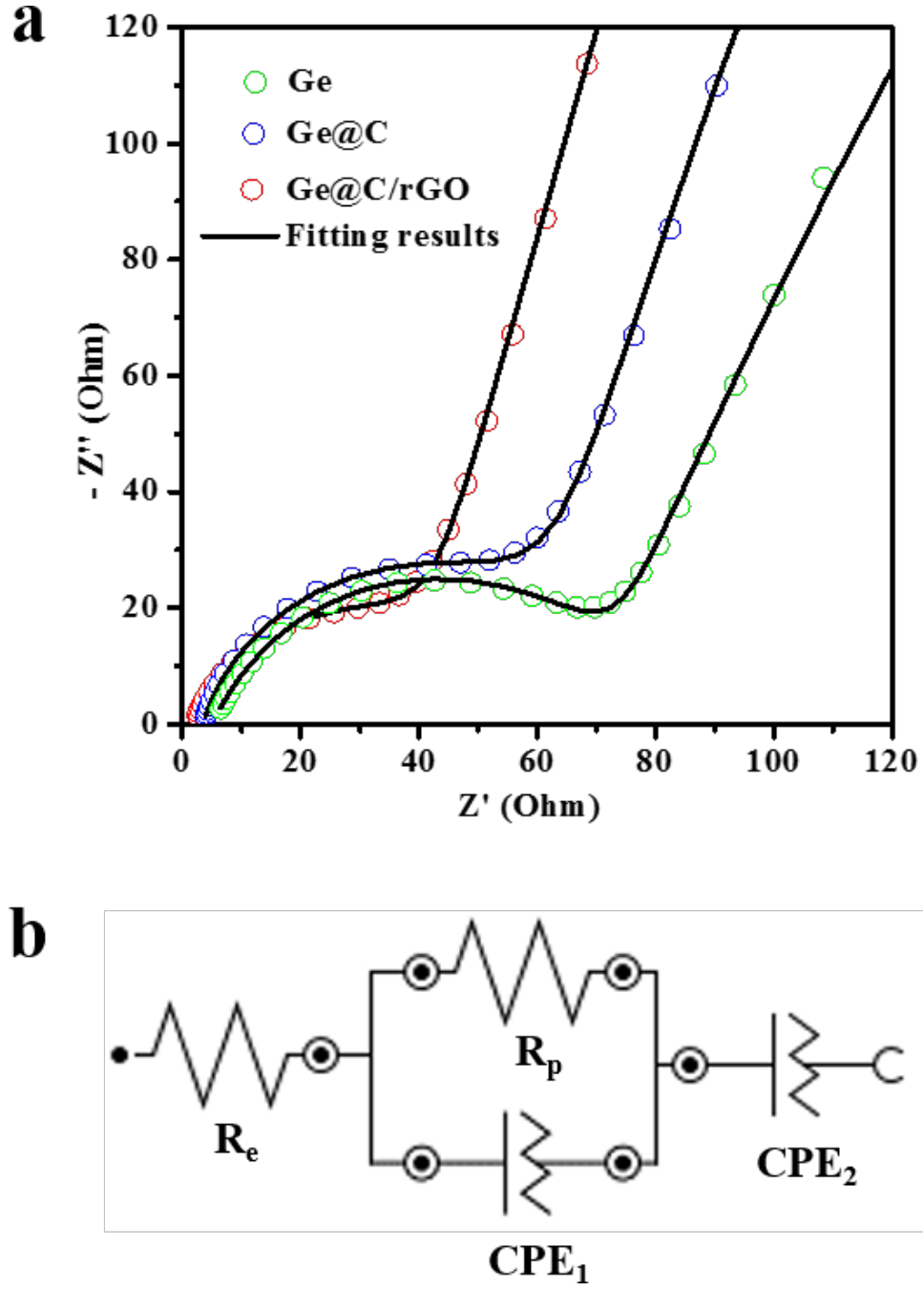

Figure 6. (a) Nyquist plots of Ge@C/rGO,Ge@C, and Ge electrodes before cycling; (b)

Equivalent circuits for Ge@C/rGO,Ge@C, and Ge electrodes used to produce fitting results.

In order to gain insight into the electrochemical performance,the electrochemical impedance spectroscopy (EIS) and corresponding equivalent circuitsbefore cycling were measured (Figure 6). Each Nyquist plot consists of a semicircle in the high-medium frequency region and a sloped line in the low frequency region. The diameter of the semicircle is due to the intrinsic electronic resistance of the materials of the anode and the low frequency sloped line before lithiation is corresponding to capacitive reactance of the anode[49, 55]. In the equivalent circuit, $R_{e}$ represents the impedance contributed by the electrolyte; $R_{p}$ represents the intrinsic electronic resistance of 
the materials. CPE (constant phase angle element) is used instead of capacitance to describe the constant change of phase angles. $\mathrm{CPE}_{1}$ represents the passivated film impedance on the lithium metal surface. $\mathrm{CPE}_{2}$ is the capacitive reactance of the anode. The value $\mathrm{R}_{\mathrm{p}}$ of $\mathrm{Ge} @ \mathrm{C} / \mathrm{rGO}$

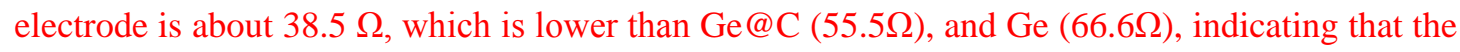
intrinsic electronic resistance of Ge@C/rGO electrode is the lowest among three different electrodes. It demonstrates that Ge@C/rGO electrode possesses highest electronic conductivity, which is due to the function of amorphous carbon/graphene double strategy, similar to the phenomenon found in germanium-carbon composites[25]. In addition, we represent the EIS measurements on fully lithiated (after 100 cycles) cellswith Ge@C/rGO and Ge@C electrodes (Figure S9).In the equivalent circuit, $\mathrm{R}_{\mathrm{e}}$ represents the impedance contributed by the electrolyte; $\mathrm{R}_{\mathrm{f}}$ and $\mathrm{CPE}_{1}$ represents the resistance and capacitance of the SEI films. $\mathrm{R}_{\mathrm{ct}}$ and $\mathrm{CPE}_{2}$ are the charge-transfer resistance and the double-layer capacitance[56-58]. The corresponding accurately values are listed in Table S1. It is obvious that the $\mathrm{R}_{\mathrm{e}}$ of both Ge@C and Ge@C/rGO is 5.75 and $4.29 \Omega$, respectively, which are very close, indicating the consistency of electrodes. The $\mathrm{R}_{\mathrm{f}}$ of

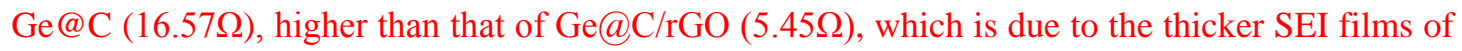
Ge@C. The $\mathrm{R}_{\mathrm{ct}}$ of both Ge@C and Ge@C/rGO is 5.49 and $4.96 \Omega$, respectively, showing similar charge transfer resistance in Ge@C and Ge@C/rGO.

We believe that the outstanding electrochemical performance of the Ge@C/rGO is attributed to the unique double carbon protection strategy. Firstly, in the core-shell structured Ge@C composite, homogeneously and continuous carbon layers can not only buffer the large volume changes of germanium, but can also improve the electronic conductivity of germanium electrode in the discharging-charging process. Secondly, core-shell structured Ge@C particles are uniformly 
and strongly anchored onto the surface of rGO nanosheets through the adhesion of dopamine, which can reduce the agglomeration of Ge@C composites and improve the utilization of active materials. Thirdly, the high flexible rGO nanosheets can weaken the stress that resulting from the repeatedvolume change and improve the structural stability of electrode during cycling. Moreover, high electrical graphene nanosheets and carbon shells form a hybrid conductive matrix, which not onlycan improve the electronic conductivity butcan also minimize the volume change ofgermanium to some extent. As a consequence, the excellent electrochemical performance of Ge@C/rGO hybrids is mainly due to the carbon/graphene double protection strategy for germanium anodes.

\section{Conclusions}

In summary, we have found an effective and novelstrategy for synthesizingthe Ge@C/rGO hybrids, generating a double carbon protection for germanium by amorphous carbon and graphene nanosheets. The key to the strategy is anchoring the Ge@C particles on therGO nanosheets through the strong adhesion of dopamine (a mussel-inspired process). The as-obtained Ge@C/rGO hybrid exhibited a reversible capacity of $1074.4 \mathrm{mAh} \mathrm{g}^{-1}$ at 2C after 600 cycles (with capacity retention of $96.5 \%$ ) and a rate capability of $436 \mathrm{mAh} \mathrm{g}^{-1}$ at 20C after 200 cycles. The excellent electrochemical performance is supposed to result from the protective, conductive and buffering effects of the carbon/graphene double carbon protection for germanium, which also confirms the successful design of Ge@C/rGO hybrids.

\section{Acknowledgements}

This work was financially supported by the National Science Foundation of China (NFSC) project No. 51432010 and JCKY2016130B010.Prof. B. V. R. Chowdari (School of Materials 
Science and Engineering,Nanyang Technological University) is greatly acknowledged for helpful discussion.

\section{Reference}

[1] M. Armand, J.M. Tarascon, Building better batteries, Nature, 451 (2008) 652-657.

[2] B.A. Korgel, Nanomaterials developments for higher-performance lithium ion batteries, J. Phys. Chem. Lett., 5 (2014) 749-750.

[3] J.S. Chen, L.A. Archer, X. Wen Lou, $\mathrm{SnO}_{2}$ hollow structures and $\mathrm{TiO}_{2}$ nanosheets for lithium-ion batteries, J. Phys. Chem. C, 21 (2011) 9912.

[4] X. Zhong, J. Wang, W. Li, X. Liu, Z. Yang, L. Gu, Y. Yu, Facile synthesis of germanium-reduced graphene oxide composite as anode for high performance lithium-ion batteries, RSC Adv., 4 (2014) 58184-58189.

[5] K.T. Lee, J.C. Lytle, N.S. Ergang, S.M. Oh, A. Stein, Synthesis and rate performance of monolithic macroporous carbon electrodes for lithium-ion secondary batteries, Adv. Funct. Mater., 15 (2005) 547-556.

[6] T. Song, Y. Jeon, M. Samal, H. Han, H. Park, J. Ha, D.K. Yi, J.-M. Choi, H. Chang, Y.-M. Choi, U. Paik, A Ge inverse opal with porous walls as an anode for lithium ion batteries, Energy Environ. Sci., 5 (2012) 9028.

[7] J. Cheng, J. Du, Facile synthesis of germanium-graphene nanocomposites and their application as anode materials for lithium ion batteries, CrystEngComm, 14 (2012) 397-400.

[8] M. Pharr, Y.S. Choi, D. Lee, K.H. Oh, J.J. Vlassak, Measurements of stress and fracture in germanium electrodes of lithium-ion batteries during electrochemical lithiation and delithiation, J. Power Sources, 304 (2016) 164-169. 
[9] M. Oh, S. Na, C.S. Woo, J.H. Jeong, S.S. Kim, A. Bachmatiuk, M.H. Rümmeli, S. Hyun, H.J. Lee, Observation of electrochemically driven elemental segregation in a si alloy thin - film anode and its effects on cyclic stability for Li - ion batteries, Adv. Energy Mater., 5 (2015).

[10] K.C. Klavetter, J.P. de Souza, A. Heller, C.B. Mullins, High tap density microparticles of selenium-doped germanium as a high efficiency, stable cycling lithium-ion battery anode material, J. Mater. Chem. A, 3 (2015) 5829-5834.

[11] N. Mahmood, T. Tang, Y. Hou, Nanostructured anode materials for lithium ion batteries: Progress, challenge and perspective Adv. Energy Mater., (2016) 1600374.

[12] C.-M. Park, J.-H. Kim, H. Kim, H.-J. Sohn, Li-alloy based anode materials for Li secondary batteries, Chem. Soc. Rev., 39 (2010) 3115-3141.

[13] C.K. Chan, X.F. Zhang, Y. Cui, High capacity Li ion battery anodes using Ge nanowires, Nano Lett., 8 (2008) 307-309.

[14] C.S. Fuller, J.C. Severiens, Mobility of Impurity Ions in Germanium and Silicon, Phys. Rev., 96 (1954) 21-24

[15] K.H. Seng, M.H. Park, Z.P. Guo, H.K. Liu, J. Cho, Self-assembled germanium/carbon nanostructures as high-power anode material for the lithium-ion battery, Angew. Chem., Int. Ed., 124 (2012) 5755-5759.

[16] G. Cui, L. Gu, L. Zhi, N. Kaskhedikar, P.A. van Aken, K. Müllen, J. Maier, A germanium-carbon nanocomposite material for lithium batteries, Adv. Mater., 20 (2008) 3079-3083.

[17] M.-H. Seo, M. Park, K.T. Lee, K. Kim, J. Kim, J. Cho, High performance Ge nanowire anode sheathed with carbon for lithium rechargeable batteries, Energy Environ. Sci., 4 (2011) 425-428. 
[18] M.-H. Seo, M. Park, K.T. Lee, K. Kim, J. Kim, J. Cho, High performance Ge nanowire anode sheathed with carbon for lithium rechargeable batteries, Energy Environ. Sci., 4 (2011) 425-428.

[19] M.H. Park, Y. Cho, K. Kim, J. Kim, M. Liu, J. Cho, Germanium Nanotubes Prepared by Using the Kirkendall Effect as Anodes for High-Rate Lithium Batteries, Angew. Chem., Int. Ed., 123 (2011) 9821-9824.

[20] M.H. Park, K. Kim, J. Kim, J. Cho, Flexible dimensional control of high-capacity Li-ion-battery anodes: From 0D hollow to 3D porous germanium nanoparticle assemblies, Adv. Mater., 22 (2010) 415-418.

[21] D.-J. Xue, S. Xin, Y. Yan, K.-C. Jiang, Y.-X. Yin, Y.-G. Guo, L.-J. Wan, Improving the electrode performance of Ge through Ge@C core-shell nanoparticles and graphene networks, J. Am. Che. Soc., 134 (2012) 2512-2515.

[22] D.T. Ngo, H.T. Le, C. Kim, J.-Y. Lee, J.G. Fisher, I.-D. Kim, C.-J. Park, Mass-scalable synthesis of 3D porous germanium-carbon composite particles as an ultra-high rate anode for lithium ion batteries, Energy Environ. Sci., 8 (2015) 3577-3588.

[23] D. Li, K.H. Seng, D. Shi, Z. Chen, H.K. Liu, Z. Guo, A unique sandwich-structured C/Ge/graphene nanocomposite as an anode material for high power lithium ion batteries, J. Mater. Chem. A, 1 (2013) 14115-14121.

[24] S. Li, C. Chen, K. Fu, R. White, C. Zhao, P.D. Bradford, X. Zhang, Nanosized Ge@CNF, Ge@C@CNF and Ge@CNF@C composites via chemical vapour deposition method for use in advanced lithium-ion batteries, J. Power Sources, 253 (2014) 366-372.

[25] T. Qiang, J. Fang, Y. Song, Q. Ma, M. Ye, Z. Fang, B. Geng, Ge@C core-shell nanostructures for improved anode rate performance in lithium-ion batteries, RSC Adv., 5 (2015) 17070-17075. 
[26] F.-W. Yuan, H.-Y. Tuan, Scalable solution-grown high-germanium-nanoparticle-loading graphene nanocomposites as high-performance lithium-ion battery electrodes: an example of a graphene-based platform toward practical full-cell applications, Chem. Mater., 26 (2014) 2172-2179.

[27] S. Xin, Y.-G. Guo, L.-J. Wan, Nanocarbon networks for advanced rechargeable lithium batteries, Acc. Chem. Res., 45 (2012) 1759-1769.

[28] K. Lu, J. Xu, J. Zhang, B. Song, H. Ma, General preparation of three-dimensional porous metal oxide foams coated with nitrogen-doped carbon for enhanced lithium storage, ACS Appl. Mater. Interfaces, (2016).

[29] J.Z. Guo, X.L. Wu, F. Wan, J. Wang, X.H. Zhang, R.S. Wang, A superior $\mathrm{Na}_{3} \mathrm{~V}_{2}\left(\mathrm{PO}_{4}\right)_{3}{ }^{-}$ based nanocomposite enhanced by both $\mathrm{N}$ - doped coating carbon and graphene as the cathode for sodium - ion batteries, Chem. - Eur. J., 21 (2015) 17371-17378.

[30] W. Li, M. Li, Z. Yang, J. Xu, X. Zhong, J. Wang, L. Zeng, X. Liu, Y. Jiang, X. Wei, L. Gu, Y. Yu, 15 Carbon-Coated Germanium Nanowires on Carbon Nanofibers as Self-Supported Electrodes for Flexible Lithium-Ion Batteries, Small, 11 (2015) 2762-2767.

[31] X. Yang, L. Zhang, F. Zhang, Y. Huang, Y. Chen, Sulfur-infiltrated graphene-based layered porous carbon cathodes for high-performance lithium-sulfur batteries, ACS Nano, 8 (2014) 5208-5215.

[32] K. Lu, D. Li, X. Gao, H. Dai, N. Wang, H. Ma, An advanced aqueous sodium-ion supercapacitor with a manganous hexacyanoferrate cathode and a $\mathrm{Fe}_{3} \mathrm{O}_{4} / \mathrm{rGO}$ anode, J. Mater. Chem. A, 3 (2015) 16013-16019.

[33] C. Lei, F. Han, D. Li, W.C. Li, Q. Sun, X.Q. Zhang, A.H. Lu, Dopamine as the coating agent 
and carbon precursor for the fabrication of $\mathrm{N}$-doped carbon coated $\mathrm{Fe}_{3} \mathrm{O}_{4}$ composites as superior lithium ion anodes, Nanoscale, 5 (2013) 1168-1175.

[34] Q. Zhu, Q. Pan, Mussel-inspired direct immobilization of nanoparticles and application for oil-water separation, ACS Nano, 8 (2014) 1402-1409.

[35] D. Li, Y. Zhang, L. Li, F. Hu, H. Yang, C. Wang, Q. Wang, Polydopamine directed MnO@C microstructures as electrode for lithium ion battery, Sci. China: Chem., 59 (2015) 122-127.

[36] Y. Bie, J. Yang, X. Liu, J. Wang, Y. Nuli, W. Lu, Polydopamine wrapping silicon cross-linked with polyacrylic acid as high-performance anode for lithium-ion batteries, ACS Appl. Mater. Interfaces, 8 (2016) 2899-2904.

[37] J. Sedo, J. Saiz-Poseu, F. Busque, D. Ruiz-Molina, Catechol-based biomimetic functional materials, Adv. Mater., 25 (2013) 653-701.

[38] Z. Liu, X.Y. Yu, U. Paik, Etching - in - a - box: A novel strategy to synthesize unique yolk shelled $\mathrm{Fe}_{3} \mathrm{O}_{4} @$ carbon with an ultralong cycling life for lithium storage, Adv. Energy Mater., (2016).

[39] L. Lin, Q. Pan, $\mathrm{ZnFe}_{2} \mathrm{O}_{4} @ \mathrm{C} /$ graphene nanocomposites as excellent anode materials for lithium batteries, J. Mater. Chem. A, 3 (2015) 1724-1729.

[40] W.S. Hummers Jr, R.E. Offeman, Preparation of graphitic oxide, J. Am. Che. Soc., 80 (1958) 1339-1339.

[41] L. Yang, Q. Gao, L. Li, Y. Tang, Y. Wu, Mesoporous germanium as anode material of high capacity and good cycling prepared by a mechanochemical reaction, Electrochem. Commun., 12 (2010) 418-421.

[42] A. Lugstein, M. Mijic, T. Burchhart, C. Zeiner, R. Langegger, M. Schneider, U. Schmid, E. 
Bertagnolli, In situ monitoring of Joule heating effects in germanium nanowires by mu-Raman spectroscopy, Nanotechnology, 24 (2013) 065701.

[43] N. Shirahata, D. Hirakawa, Y. Masuda, Y. Sakka, Size-dependent color tuning of efficiently luminescent germanium nanoparticles, Langmuir, 29 (2013) 7401-7410.

[44] Y. Xiao, X. Wang, W. Wang, D. Zhao, M. Cao, Engineering hybrid between MnO and N-doped carbon to achieve exceptionally high capacity for lithium-ion battery anode, ACS Appl. Mater. Interfaces, 6 (2014) 2051-2058.

[45] C. Hu, Y. Xiao, Y. Zhao, N. Chen, Z. Zhang, M. Cao, L. Qu, Highly nitrogen-doped carbon capsules: scalable preparation and high-performance applications in fuel cells and lithium ion batteries, Nanoscale, 5 (2013) 2726-2733.

[46] C. Zhang, Z. Lin, Z. Yang, D. Xiao, P. Hu, H. Xu, Y. Duan, S. Pang, L. Gu, G. Cui, Hierarchically designed germanium microcubes with high initial coulombic efficiency toward highly reversible lithium storage, Chem. Mater., 27 (2015) 2189-2194.

[47] Y. Yu, C. Yan, L. Gu, X. Lang, K. Tang, L. Zhang, Y. Hou, Z. Wang, M.W. Chen, O.G. Schmidt, J. Maier, Three-dimensional (3D) bicontinuous Au/amorphous-Ge thin films as fast and high-capacity anodes for lithium-ion batteries, Adv. Energy Mater., 3 (2013) 281-285.

[48] F.-W. Yuan, H.-J. Yang, H.-Y. Tuan, Alkanethiol-passivated Ge nanowires as high-performance anode materials for lithium-ion batteries: the role of chemical surface functionalization, ACS Nano, 6 (2012) 9932-9942.

[49] Y.C. Zhang, Y. You, S. Xin, Y.X. Yin, J. Zhang, P. Wang, X.S. Zheng, F.F. Cao, Y.G. Guo, Rice husk-derived hierarchical silicon/nitrogen-doped carbon/carbon nanotube spheres as low-cost and high-capacity anodes for lithium-ion batteries, Nano Energy, 25 (2016) 120-127. 
[50] B. Luo, B. Wang, X. Li, Y. Jia, M. Liang, L. Zhi, Graphene - confined Sn nanosheets with enhanced lithium storage capability, Adv. Mater., 24 (2012) 3538-3543.

[51] X. Wang, X. Cao, L. Bourgeois, H. Guan, S. Chen, Y. Zhong, D.M. Tang, H. Li, T. Zhai, L. Li, $\mathrm{N}$ - doped graphene - $\mathrm{SnO}_{2}$ sandwich paper for high - performance lithium - ion batteries, Adv. Funct. Mater., 22 (2012) 2682-2690.

[52] C. Zhang, X. Peng, Z. Guo, C. Cai, Z. Chen, D. Wexler, S. Li, H. Liu, Carbon-coated $\mathrm{SnO}_{2} /$ graphene nanosheets as highly reversible anode materials for lithium ion batteries, Carbon, 50 (2012) 1897-1903.

[53] A.M. Chockla, K.C. Klavetter, C.B. Mullins, B.A. Korgel, Solution-grown germanium nanowire anodes for lithium-ion batteries, ACS Appl. Mater. Interfaces, 4 (2012) 4658-4664.

[54] T. Kennedy, E. Mullane, H. Geaney, M. Osiak, C. O'Dwyer, K.M. Ryan, High-performance germanium nanowire-based lithium-ion battery anodes extending over 1000 cycles through in situ formation of a continuous porous network, Nano Lett., 14 (2014) 716-723.

[55] J.Y. Song, H.H. Lee, Y.Y. Wang, C.C. Wan, Two- and three-electrode impedance spectroscopy of lithium-ion batteries, J. Power Sources, 111 (2002) 255-267.

[56] R. Ruffo, S.S. Hong, C.K. Chan, R.A. Huggins, Y. Cui, Impedance analysis of silicon nanowire lithium ion battery anodes, J. Phys. Chem. C, 113 (2009) 11390-11398.

[57] M. Zhang, Y. Li, E. Uchaker, S. Candelaria, L. Shen, T. Wang, G. Cao, Homogenous incorporation of $\mathrm{SnO} 2$ nanoparticles in carbon cryogels via the thermal decomposition of stannous sulfate and their enhanced lithium-ion intercalation properties, Nano Energy, 2 (2013) 769-778.

[58] J. Hassoun, P. Ochal, S. Panero, G. Mulas, C. Bonatto Minella, B. Scrosati, The effect of CoSn/CoSn2 phase ratio on the electrochemical behaviour of Sn40Co40C20 ternary alloy 
electrodes in lithium cells, J. Power Sources, 180 (2008) 568-575. 